\title{
The third-order law for magnetohydrodynamic turbulence with constant shear
}

\author{
M. Wan*, S. Oughton ${ }^{\dagger}$, S. Servidio* and W. H. Matthaeus* \\ *Bartol Research Institute and Department of Physics and Astronomy, University of Delaware, Newark, \\ Delaware 19716, USA \\ ${ }^{\dagger}$ Department of Mathematics, University of Waikato, Hamilton 3240, New Zealand
}

\begin{abstract}
The scaling laws of mixed third-order structure functions for isotropic, homogeneous, and incompressible magnetohydrodynamic (MHD) turbulence have been recently applied in solar wind studies, even though there is recognition that isotropy is not well satisfied. Other studies have taken account of the anisotropy induced by a constant mean magnetic field. However, large-scale shear can also cause departures from isotropy. Here we examine shear effects in the simplest case, and derive the third-order laws for MHD turbulence with constant shear, where homogeneity is still assumed. This generalized scaling law has been checked by data from direct numerical simulations (DNS) of two-dimensional (2D) MHD and is found to hold across the inertial range. These results suggest that third-order structure function analysis and interpretation in the solar wind should be undertaken with some caution, since, when present, shear can change the meaning of the third-order relations.
\end{abstract}

\section{INTRODUCTION}

The so-called four-fifths law for the third-order structure function $[1,2]$ is a central relationship in turbulence theory, and has been widely used in characterizing the turbulent energy cascade in incompressible hydrodynamics [3]. This similarity law identifies the inertial range of the energy cascade, where one finds that

$$
\left\langle\left(\delta u_{L}(\mathbf{x}, \mathbf{r})\right)^{3}\right\rangle=-\frac{4}{5} \varepsilon r
$$

with $\delta u_{L}(\mathbf{x}, \mathbf{r})=[\mathbf{u}(\mathbf{x}+\mathbf{r})-\mathbf{u}(\mathbf{x})] \cdot \hat{\mathbf{r}}$ the longitudinal velocity increment along the spatial separation $\mathbf{r}$, and $\langle\ldots\rangle$ indicating an ensemble average. The constant $\varepsilon$ is the mean energy dissipation rate, and is assumed to remain finite as the Reynolds number increases without bound [4].

In magnetohydrodynamic (MHD) the analog of the 4/5-law was derived by Politano and Pouquet [5, 6]. They obtained symmetric scaling laws for each of the Elsässer fields $\mathbf{z}^{ \pm}=\mathbf{v} \pm \mathbf{b}$. Under the assumptions of full isotropy and homogeneity, the mixed third-order structure function relations in MHD are [5-7]:

$$
\delta z_{L}^{\mp} \delta z_{i}^{ \pm 2}=-\frac{4}{d} \varepsilon^{ \pm} r
$$

where $d$ is the dimension, $\delta \mathbf{z}^{ \pm}=\mathbf{z}^{ \pm}(\mathbf{x}+\mathbf{r})-\mathbf{z}^{ \pm}(\mathbf{x})$ are the increments of the Elsässer variables, and $\varepsilon^{ \pm}$are the corresponding mean energy dissipation rates of $\mathbf{z}^{ \pm}$. Recently, MHD third-order laws have been applied in solar wind studies, with the aim of measuring the energy cascade rate. These studies either continue to assume isotropy [7, 8], or deal with anisotropy caused by a constant mean magnetic field [9-11].

An essential point is that homogeneity, timestationarity and isotropy must be satisfied, in some form [12], in order to arrive at the law in the above form of Eqs. (1) and (2). These constraints are rarely verified in nature, so a generalization of this important law of turbulence is needed. Regarding isotropy, in the hydrodynamic case Casciola et al. [13] extended the 4/5-law to shear-dominated flows, where additional terms enter the balance between dissipation and the third-order structure function. This can have an interesting counterpart in the MHD case. For example, in solar wind applications, large-scale shear can be present (in connection for example with the interfaces between high-speed and low-speed streams [14]). It is of broad interest to examine how the presence of such shear might modify the law given by Eq. (2) in stationary turbulence. Here we take a preliminary step in that direction by examining the MHD law for an anisotropic but steady and homogeneous medium with a uniform constant specified shear in the velocity field [15], and then we verify the new law using direct numerical simulations of turbulence.

\section{FORMULATION}

Following procedures analogous to those used to obtain the isotropic MHD 4/5-law [5-7], and the hydrodynamic extension to the 4/5-law for constant shear [13], we obtain an MHD third-order law with constant shear of velocity [16]. Let us assume a decomposition of the 
velocity field $\mathbf{v}=\mathbf{U}+\mathbf{u}$ into a mean velocity $\mathbf{U}(\mathbf{x})$ and a fluctuating component $\mathbf{u}(\mathbf{x}, t)$. We arrive at the modified third-order law for MHD in integral form:

$$
\begin{aligned}
& \oint\left[\left\langle\left(\delta z_{k}^{\mp}+\delta U_{k}\right)\left|\delta z_{i}^{ \pm}\right|^{2}\right\rangle n_{k}\right] \mathrm{d} S_{r}+2 \frac{\partial U_{i}}{\partial x_{k}} \int\left\langle\delta z_{i}^{ \pm} \delta z_{k}^{\mp}\right\rangle \mathrm{d} V_{r} \\
& =-\frac{16 \pi r^{3}}{3} \varepsilon^{ \pm} .
\end{aligned}
$$

The volume integral is over a sphere of radius $r\left(V_{r}, S_{r}\right.$ are the volume and surface), and $n_{k}$ is a unit vector normal to the surface. By setting $\mathbf{U}=\mathbf{0}$, Eq. (3) will recover the standard third-order law for isotropic MHD turbulence [5], given by Eq. (2).

We now adapt the above MHD law to the case of 2D geometry, to improve the statistical significance of our numerical results. Moreover, a fully 3D treatment would necessarily have a less extensive inertial range.

Let us now define in Eq. (3) $P_{3}^{ \pm}(\mathbf{r})=\left\langle\delta z_{k}^{\mp}\left|\delta z_{i}^{ \pm}\right|^{2}\right\rangle r_{k} / r$, where $\mathbf{r}$ is the separation vector. For isotropic MHD, we will have $P_{3}^{ \pm}(r)=\left\langle\delta z_{r}^{\mp}\left|\delta z_{i}^{ \pm}\right|^{2}\right\rangle$, and then Eq. (3) simplifies to

$$
P_{3}^{ \pm}(r)=-2 \varepsilon^{ \pm} r
$$

which is the 2D version of the third-order law Eq. (2).

For anisotropic 2DMHD with a constant solenoidal shear, that we impose in the $x$ direction, $\alpha=\frac{\mathrm{d} U_{y}(x)}{\mathrm{d} x}$. In this case, Eq. (3) can be simplified as:

$$
\begin{aligned}
& \int_{0}^{2 \pi} P_{3}^{ \pm}(\mathbf{r}) \pi r \mathrm{~d} \theta+\int_{0}^{2 \pi} \delta U_{r}\left\langle\left|\delta z_{i}^{ \pm}\right|^{2}\right\rangle \pi r \mathrm{~d} \theta \\
& +2 \alpha \int_{0}^{r} \int_{0}^{2 \pi}\left\langle\delta z_{x}^{ \pm} \delta z_{x}^{\mp}\right\rangle \pi r^{\prime} \mathrm{d} \theta \mathrm{d} r^{\prime}=-4 \pi r^{2} \varepsilon^{ \pm},(5)
\end{aligned}
$$

where $\theta$ is the angle $\mathbf{r}$ makes with the the $x$ axis and $\delta U_{r}=\delta \mathbf{U}(\mathbf{r}) \cdot \hat{\mathbf{r}}$. We can then define:

$$
\begin{gathered}
S^{ \pm}=-\frac{1}{2 \pi} \int_{0}^{2 \pi} P_{3}^{ \pm}(\mathbf{r}) \mathrm{d} \theta \\
S_{U}^{ \pm}=-\frac{1}{2 \pi} \int_{0}^{2 \pi} \delta U_{r}\left\langle\left|\delta z_{i}^{ \pm}\right|^{2}\right\rangle \mathrm{d} \theta, \\
S_{\alpha}^{ \pm}=-\frac{\alpha}{\pi r} \int_{0}^{r} \int_{0}^{2 \pi}\left\langle\delta z_{y}^{ \pm} \delta z_{x}^{\mp}\right\rangle r^{\prime} \mathrm{d} \theta \mathrm{d} r^{\prime} .
\end{gathered}
$$

In terms of Eq. (6)-(8), Eq. (5) can be rewritten as:

$$
S^{ \pm}+S_{U}^{ \pm}+S_{\alpha}^{ \pm}=2 \varepsilon^{ \pm} r
$$

The second term $S_{U}^{ \pm}$is similar to the ordinary third-order structure function $S^{ \pm}$, but involves the shear, and $S_{\alpha}^{ \pm}$is another term that involves the shear and the second-order structure function of the fluctuations.

To test how the modified third-order law is influenced by these new terms, we now carry out an examination below using turbulence simulation data.

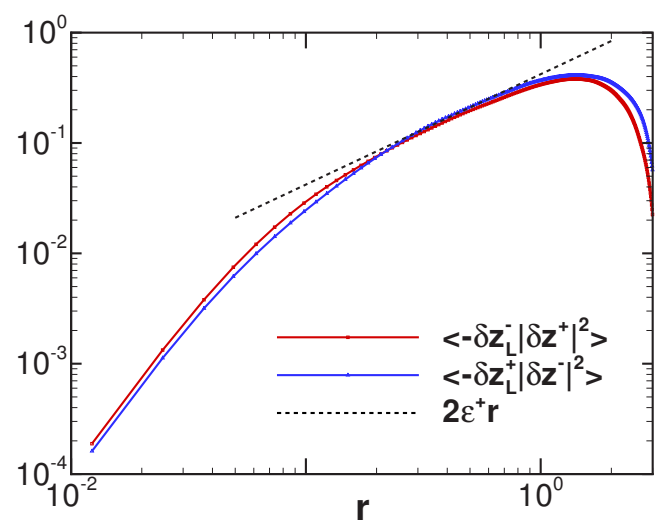

FIGURE 1. The scaling of mixed third-order structure functions for the isotropic MHD simulation.

\section{NUMERICAL SIMULATIONS}

We perform direct numerical simulations of turbulence in order to test the validity of Eq. (9). We numerically simulated forced 2D incompressible MHD turbulence. The specific approach we use is to solve the following modified equations for the vector potential $a$ and vorticity $\omega$ :

$$
\begin{array}{r}
\partial_{t} \omega+(\mathbf{v}+\mathbf{U}) \cdot \nabla(\omega+\Omega)=\mathbf{b} \cdot \nabla j+v \nabla^{2}(\omega+\Omega)+F_{\omega}, \\
\partial_{t} a+(\mathbf{v}+\mathbf{U}) \cdot \nabla a=\eta \nabla^{2} a+F_{a},
\end{array}
$$

where the magnetic field $\mathbf{b}=\nabla a \times \hat{\mathbf{z}}$, the current density $j=-\nabla^{2} a$. The velocity is given by $\mathbf{v}=\nabla \psi \times \hat{\mathbf{z}}$, where $\nabla^{2} \psi=-\omega$. The specified shear velocity is $\mathbf{U}=U_{y}(x) \hat{y}$ and its associated vorticity $\Omega=\nabla \times \mathbf{U}$ are constant in time. Time $t$ is in units of nominal large scale nonlinear or eddy turnover times. A Fourier pseudo-spectral method with periodic boundary conditions is used. The time-dependent magnetic forcing $F_{a}$ is imposed by constraining the magnetic energy in the first two (lowest) wavenumber shells of $\mathbf{b}$ at constant level; the forcing $F_{\omega}$ on the velocity is imposed also by constraining the energy in the first two wavenumber shells of $\mathbf{v}$, but the forcing is only in the perpendicular direction relative to the magnetic field, a condition that can be enforced at each wavevector. For example, $\tilde{F}_{\omega}(\mathbf{k}) \cdot \tilde{\mathbf{a}}(\mathbf{k})=\mathbf{0}$, so that the cross helicity will be kept small, where . means a quantity in the Fourier space.

\section{Isotropic MHD turbulence}

First we simulate the 2DMHD system without the presence of the shear, imposing simply $\mathbf{U}=\mathbf{0}$ in Eqs. (10)-(11), with the parameters listed in Table 1.

In order to measure this inertial range, and extract the mean energy transfer from the fluctuations, we analyzed 
TABLE 1. Parameters for the isotropic $(\alpha=0)$ and anisotropic simulations.

\begin{tabular}{rrrrr}
\hline & Resolution & $v(=\eta)$ & $\varepsilon^{ \pm}$ & $\alpha$ \\
\hline Run 1 & $1024^{2}$ & 0.001 & 0.21 & 0 \\
Run 2 & $2048^{2}$ & 0.0006 & 0.084 & 1.57 \\
\hline
\end{tabular}

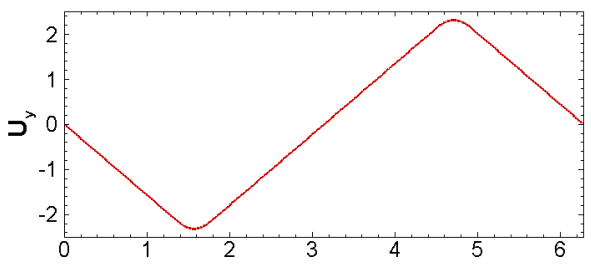

FIGURE 2. Shear velocity profile $U_{y}(x)$.

the classical mixed third-order structure functions. In Fig. 1 we show the scaling of $P_{3}^{ \pm}(r)$. In some range, both functions scale $\propto r$. The results are consistent with the numerical results reported in [17]. From Eq. (4), $\varepsilon^{ \pm}$can be extracted from the scaling of $P_{3}^{ \pm}(r)$, that is $\varepsilon^{ \pm}=-P_{3} /(2 r)$. Based on our data in Fig. 1, we obtain $\varepsilon^{+}=0.21$ and $\varepsilon^{-}=0.22$. Comparing these values with the definition of the dissipation rates, $\varepsilon^{ \pm}=v\left\langle\left|\partial_{k} z_{i}^{ \pm}\right|^{2}\right\rangle$, a good agreement is found.

\section{Anisotropic MHD turbulence with shear}

We want to test the generalization of the MHD thirdorder law, described in Eqs. (6)-(9), by examining turbulence that evolves in the presence of a constant shear. To enable a numerical experiment of this type that employs an accurate and well-tested pseudo-spectral method (same as the isotropic case described before), we adopt the following strategy. We simulate 2D MHD turbulence in periodic geometry, but impose a large-scale velocity that is characterized by large regions of nearly constant shear. In particular we impose the constant velocity $U_{y}(x)$; the particular form is plotted in Fig. 2. The constant shear occurs in two regions with shear rate $\alpha$ and $-\alpha$, where $\alpha \approx 1.57$. Two buffer regions between the shear flows are present, where the imposed shear changes sign. The parameters for the simulation are listed in Table 1.

In Fig. 3, we show the scaling of $S^{+}, S_{U}^{+}$and $S_{\alpha}^{+}$using data from the positive shear region, where $2 \varepsilon^{+} r$ is plotted for comparison. Although we can observe a short range where $S^{+}$scales $\propto r$, this range is much smaller than the isotropic case (see Fig. 1). If one was to attempt to use this linear scaling of $S^{+}$as a substitute for the full modified third-order law Eq. (3), there is no assurance

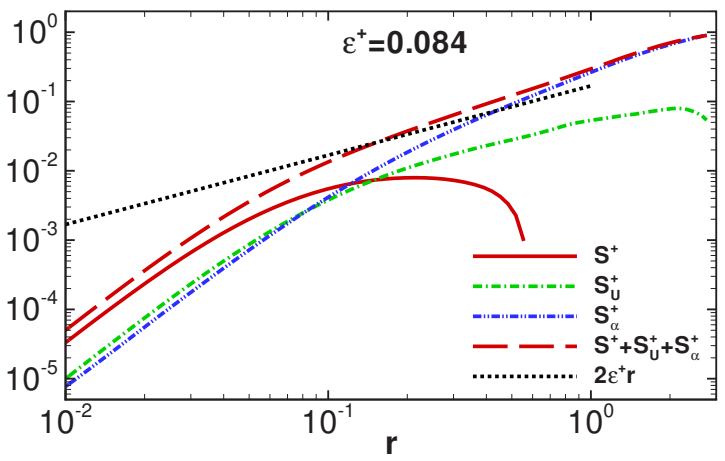

FIGURE 3. The scaling of the individual third-order terms $S^{+}, S_{U}^{+}, S_{\alpha}^{+}$, and their sum for a positive shear region.

that the result would be relevant to the actual dissipation rate. (However, one might be tempted to carry out this procedure if, for example, this were experimental data and the experimenter were unaware of the presence of the shear, and therefore assumed that Eq. (2) was valid.) In fact, for this particular case the dissipation extrapolated from this scaling would be $\varepsilon^{+} \approx 0.03$, which grossly underestimates the actual dissipation rate.

There is no reason that $S^{+}, S_{U}^{+}$, and $S_{\alpha}^{+}$should each have a (separate) scaling law; in fact, as obtained in Eq. (9), only the sum of these quantities obeys the scaling law. We can see evidence, in Fig. 3, that the modified third-order law produces a very good linear scaling. For the sum of the three terms, we get a much longer range of scaling $\propto r$ (as compared to any individual term), and the dissipation extracted from this scaling is $\varepsilon^{+} \approx 0.1$, which just slightly overestimates the actual dissipation.

\section{SUMMARY AND DISCUSSIONS}

We examined the mixed third-order MHD structure functions, incorporating a constant sheared velocity in addition to homogeneous fluctuations, under a set of assumptions that parallels those used in standard turbulence theory to derive the Kolmogorov 4/5-law. In analogy to the findings of Casciola [13] and Lindborg [18] for hydrodynamics, we find that a law can be obtained for stationary homogenous turbulence that relates third-order structure functions and dissipation, but which also involves additional terms. For MHD with imposed constant shear, there are new, additional terms that appear in this modified third-order law, as in the hydrodynamic case.

We have employed 2D simulations to demonstrate the modified law, a strategy designed to enable high statistical weight tests to be done using an accurate pseudospectral code. We found that the modified relationship $S^{ \pm}+S_{U}^{ \pm}+S_{\alpha}^{ \pm}=2 \varepsilon^{ \pm} r$ is well satisfied. The sum of the 
three structure function terms scales linearly with separation in the inertial range.

However, as indicated in [7], isotropy is not fully satisfied in the solar wind. Anisotropic MHD turbulence, arising, for example, due to the presence of a strong uniform magnetic field [19] has attracted wide interest in the context of the solar wind [20-22]. Indeed, recent studies $[10,11]$ have also extended the treatment of the thirdorder MHD laws to a form in which spectral anisotropy can be accommodated. In particular MacBride et al. [10] model the solar wind magnetic fluctuations using both isotropic and non-isotropic model (a hybrid 1D+2D model) when they evaluate heating rates using the homogeneous third-order law. However, their formulation, and similar studies that have appeared recently (e.g., $[7,8]$ ), have so far maintained the assumption that the largescale magnetic field and plasma flows are uniform. This eliminates by assumption the possibility that large-scale shear can enter the balance between cascade and dissipation, as it does in the hydrodynamics study and its antecedents $[13,18]$.

Solar wind applications have dealt with these issues in various ways, such as simply adopting the isotropic form [7], or employing alternate symmetries, such as 2D [10] or by introducing additional conservation laws [11], or assumptions about angular variation of anisotropy [23]. The present results are complementary to these, in that the new third-order structure function relations include effects of anisotropy. However for the case of uniform shear there is the additional significant complication that the functional form of the third-order relation itself is changed.

It is unclear how extensive the implied changes to the interpretation of the third-order law might be for the solar wind. On the basis of a very simple estimate we expect the new terms in the third-order equation to be of significance when the large-scale velocity increments are of the same order or larger than the fluctuation increments at the same separation, $\delta U \sim \delta z$. For the solar wind, near a stream interface one might estimate that the large-scale velocity changes $\Delta U \approx 100 \mathrm{~km} / \mathrm{s}$ over a distance of $1 / 100$ AU. Since this is comparable to correlation scale, one can estimate that over the same distance the velocity increments are of the order of $30 \mathrm{~km} / \mathrm{s}$. In such regions, shear could be a major factor. In weaker shear regions at high latitude, microstreams could contribute $\Delta U \approx 50 \mathrm{~km} / \mathrm{s}$ over six hours, and the fluctuation increment might be of order $40 \mathrm{~km} / \mathrm{s}$ in these regions. Again, shear emerges as a possibly significant factor. On this basis we suggest caution in developing interpretations that define either cascade rates or the span of the inertial range on the basis of the observed linear behavior of the third-order mixed structure function. The present simplified constant shear third-order law indicates that additional terms, and alternative interpretations, may be needed.

This research supported in part by the NSF Solar Terrestrial Program under grant ATM0539995 and by NASA under the Heliophysics Theory Program grant NASA NNX08AI47G.

\section{REFERENCES}

1. A. N. Kolmogorov, Dokl. Akad. Nauk SSSR 30, 301-305 (1941) [Proc. R. Soc. London, Ser. A 434, 9-13 (1991)].

2. A. S. Monin and A. M. Yaglom, Statistical Fluid Mechanics II, MIT Press, Cambridge (MA), 1975.

3. U. Frisch, Turbulence: The Legacy of A. N. Kolmogorov, Cambridge (UK), 1995, 72-115.

4. T. de Kármán and L. Howarth, Proc. R. Soc. Lond. A 164, 192-215 (1938).

5. H. Politano and A. Pouquet, Geophys. Res. Lett. 25, 273-276 (1998).

6. H. Politano and A. Pouquet, Phys. Rev. E 57, R21-R24 (1998).

7. L. Sorriso-Valvo, R. Marino, V. Carbone, A. Noullez, F. Lepreti, P. Veltri, R. Bruno, B. Bavassano, and E. Pietropaolo, Phys. Rev. Lett. 99, 115001 (2007).

8. R. Marino, L. Sorriso-Valvo, V. Carbone, A. Noullez, R. Bruno, and B. Bavassano, Astrophys. J. 677, L71-L74 (2008).

9. B. T. MacBride, M. A. Forman, and C. W. Smith, Proc. Solar Wind 11 - SOHO 16, 613-616, 2005.

10. B. T. MacBride, C. W. Smith, and M. A. Forman, Astrophys. J. 679, 1644-1660 (2008).

11. J. E. Stawarz, C. W. Smith, B. J. Vasquez, M. A. Forman, and B. T. MacBride, Astrophys. J. 697, 1119-1127 (2009).

12. R. J. Hill, J. Fluid Mech. 353, 67-81 (1997).

13. C. M. Casciola, P. Gualtieri, R. Benzi, and R. Piva, J. Fluid Mech. 476, 105-114 (2003).

14. D. J. McComas, B. L. Barraclough, H. O. Funsten, J. T. Gosling, E. Santiago-Munoz, R. M. Skoug, B. E. Goldstein, M. Neugebauer, P. Riley, A. Balogh, J. Geophys. Res. 105, 10419-10434 (2000).

15. M. M. Rogers and P. Moin, J. Fluid Mech. 176, 33-66 (1987).

16. M. Wan, S. Servidio, S. Oughton, and W. H. Matthaeus, Phys. Plasmas, 16, 090703 (2009).

17. L. Sorriso-Valvo, V. Carbone, A. Noullez, H. Palitano, A. Pouquet, and P. Veltri, Phys. Plasmas 9, 89-95 (2002).

18. E. Lindborg, J. Fluid Mech. 326, 343-356 (1996).

19. S. Oughton, E. R. Priest, and W. H. Matthaeus, J. Fluid Mech. 280, 95-117 (1994).

20. J. W. Belcher and L. Davis, J. Geophys. Res. 76, 3534-3563 (1971).

21. W. H. Matthaeus, M. L. Goldstein, and D. A. Roberts, J. Geophys. Res. 95, 20673-20683 (1990).

22. J. W. Bieber, W. Wanner, and W. H. Matthaeus, J. Geophys. Res. 101, 2511-2522 (1996).

23. J. J. Podesta, M. A. Forman, C. W. Smith, D. C. Elton, Y. Malecot, and Y. Gagne, Nonlin. Processes Geophys. 16, 99-110 (2009). 